\title{
Reading Your Mind: EEG during Reading Task
}

\author{
Tan Vo and Tom Gedeon \\ School of Computer Science, The Australian National University, \\ Acton, Canberra, ACT 0200, Australia \\ \{tan.vo, tom.gedeon\}@anu.edu.au
}

\begin{abstract}
This paper demonstrates the ability to study the human reading behaviors with the use of Electroencephalography (EEG). This is a relatively new research direction because, obviously, gaze-tracking technologies are used specifically for those types of studies. We suspect, EEG, with the capability of recording brain-wave activities from the human scalp, in theory, could exhibit potential attributes to replace gaze-tracking in such research. To prove the concept, in this paper, we organized a BCI experiment and propose a model for effective classifying EEG data in comparison to the accuracy of gaze-tracking. The results show that by using EEG, we could achieve comparable results against the more established methods while demonstrating a potential live EEG applications. This paper also discusses certain points of consideration for using EEG in this work.
\end{abstract}

Keywords: BCI, Artificial Neural Network, EEG, Reading tasks, Signal Processing.

\section{Introduction}

We conducted an experiment where we capture test participants EEG activities while they perform reading tasks. We analyzed a set of EEG features such as the frequency activations of EEG alpha, theta, beta bands etc.... to identify the key factors showing user engagement level in reading. The results are analyzed for each individual participant against the whole set of participants. Our aim is to verify the following hypotheses: "For each participant, can we effectively identify the link between EEG brain activities and his level of engagement in the reading task?" and "Overall, can we achieve a general method to effectively identify the link between EEG brain activities and the level of engagement in the reading task?" For this paper, together with analyzing these hypotheses, we also propose a method of processing EEG signals that is effective enough to be considered for a real-time classification system. We use an Artificial Neural Network (ANN) technique to validate the efficiency of the proposed approach.

\section{Backgrounds}

\subsection{General}

Reading is an activity that most human today perform on a very regular basis. We read and process information so much that reading skill becomes an almost 
second nature to us. The conjecture we propose here is based on that statement. So comprehensive and comfortable are we in reading words, texts, that we would show, intentionally or not, certain behaviors that could be used to interpret our perception of the contents we read. Understanding the meaning of words in sentences and paragraphs places a certain strain on a person's cognitive process. Depends on various contexts, such strains could go unnoticed by most of us. An example of such process would be, in order to comprehend a text, a person needs to build up linkages of information that he previously obtained with the current text. That cognitive process would be more significant if the text contains more information that he would, deliberately or not, associate back to. That assumption is reasonably correct because a normal person can only keep seven pieces of information $( \pm 2)$ in their short-term memory [4. We had some success in [6] in identifying such a process using gaze-tracking technique. For this experiment, however, we would like to validate the results with EEG. Such use of EEG is a brain-computer interaction technique that monitors brain-wave activities from the human scalp. We suspect that the EEG signals would exhibit those aforementioned cognitive activities during reading tasks. A point for consideration in doing research with EEG is to deal with eye movement artifacts in EEG signals. Eye muscles produce considerable EEG signal noises and traditionally, EEG researchers would remove them from the signal analysis [8]. For this paper, however, we would like to propose a different approach to that by not eliminating the effect of eye movements from our analysis of reading tasks. Reading tasks have one unique characteristic that supports our view: a persons eye movements tie quite robustly to their engagement to the contents being read. The increase/decrease in the amount of skipping forward and back-tracking activities found in the gaze correlate with the increase/decrease of the cognitive load in reading [6]. Studying of reading without regard to eye-gaze could limit the potential outcome. As our aim is to identify the same link through the use of EEG instead of gaze-tracking technology, we would like to take advantage of this "good noise.

Another point for consideration is that the EEG signal, by nature, is stochastic. In regards to this experiment, it suggests that the 19 different participants EEG data should be processed and analyzed individually. In this paper, however, we will try both approaches: consider each participant individually vs. all participants as a whole. We then in turn compare the outcomes. We also compare the results with the gaze data we collected from our previous experiment [6].

\subsection{Preliminary Analysis}

For the initial analysis, we ran time-frequency analysis on the raw EEG data, as suggested by Makeig[7]. We would like to observe the differences in timefrequency distributions of EEG signals captured from a person reading a relevant against irrelevant piece of text (English paragraphs). This analysis is performed on the first raw EEG channel (Fp1) of each participant using Fourier Transform and the time resolution is going to be (time taken to read, in milliseconds, over 512 epochs). The initial observation has revealed that there is a lack of 
apparent and consistent features that could help distinguished the two classes. Having said that, there is a minor difference that can be spotted by observing those spectrographs - that is, there are more drops in amplitudes found on the spectrographs of EEG signals recorded from reading irrelevant paragraphs compared to the one obtained from reading relevant paragraphs.

Figure 1 demonstrates the above observation. It includes spectrograms generated by analyzing the EEG signal a participant reading two relevant paragraphs (Left-hand Side) and two irrelevant paragraphs(Right-hand side). The circled spots are some of the locations where the drops in amplitude can be identified.

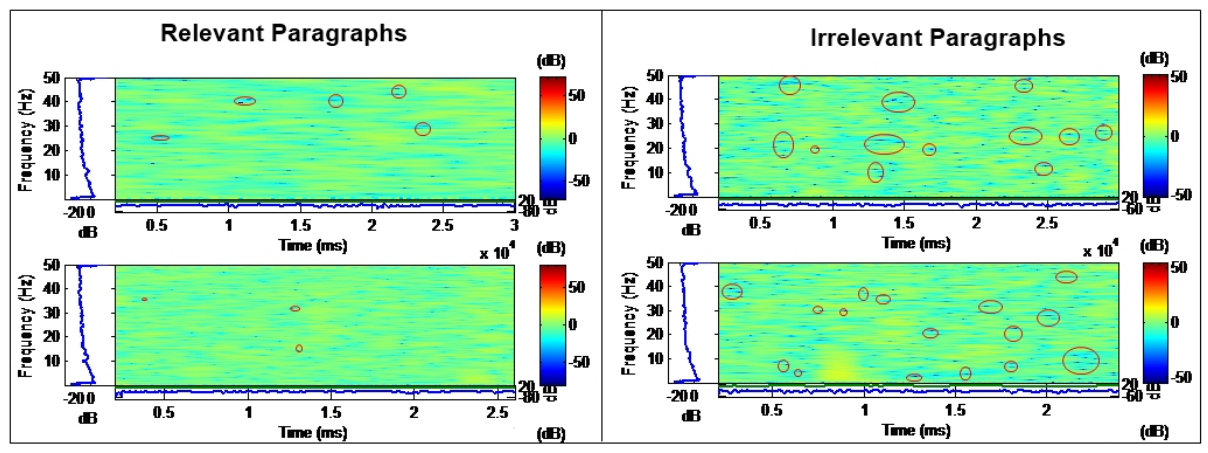

Fig. 1. A distribution of cell movement distances throughout reading activity of a paragraph

The initial analysis has showed to us that there is a possibility that, using statistical machine learning techniques, we could effectively classify the two cases. For that purpose, we are going to use a standard Artificial Neural Network configuration as the foundation. Optimization will be considered if the initial results are promising.

\section{Experiment}

We have 19 participants for this experiment. The experiment involves the participant reading some paragraphs from a computer screen while the computer captures their brain-wave activities via EEG equipment. In total there were ten paragraphs for the participants to read. Seven of the paragraphs were taken from the paper "Keyboard before Head Tracking Depresses User Success in Remote Camera Control" by Zhu et al. 1]. The remaining three paragraphs were extracts from various sources (miscellaneous paragraphs).

Five of the paragraphs from the paper were chosen for the amount of useful information that was contained within and they are relevant to each other. The other five paragraphs (two from the aforementioned paper and the three miscellaneous ones) were chosen because of their generality and lack of specific 
technical information - they are irrelevant with the other five and also are irrelevant between themselves. Care was taken to make sure that this fact was not obvious to experiment participants.

\subsection{Setups}

For each of the 19 volunteer participants, the general instructions are to read as if they were just reading any regular piece of text, and that they would not be questioned about the paragraphs read at the end of the trial. For recording EEG signals, which are very sensitive, we also paid attention to eliminate as much external distraction as possible during each trial. The experiment is designed to help show which participants could look at the bigger picture even when the information is out of sequence and scattered. Hence, the paragraphs were presented to participants in different orders to prevent any specific paragraph ordering from affecting the results. Figure 2(a) shows one of the paragraphs that each participant read. The screen for reading is about $72 \mathrm{~cm}$ away from the participant face. The head position of participant is secured with a chin rest. This is to minimize head/face movements (intentionally or not) - which could greatly affect the EEG signals. The EEG equipment we used in this experiment is BioSemi ActiveTwo. We recorded with 16 channels marked and placed according to the 10-20 system .These 16 channels are as followed: Fp1, Fp2, F4, Fz, F3, T7, C3, Cz, C4, T8, P4, Pz, P3, O1, Oz, O2. Figure 2(b) shows one participant setup with the 16 electrodes. The recording was continuously throughout the trial of

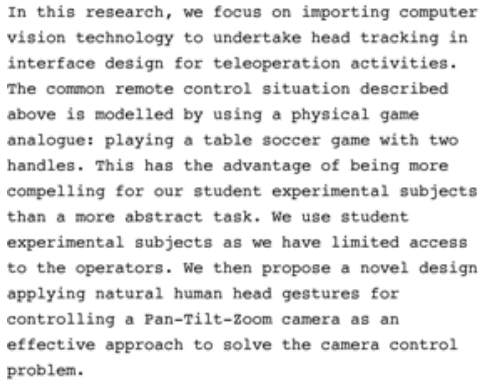
problem.

(a) One of the reading paragraphs

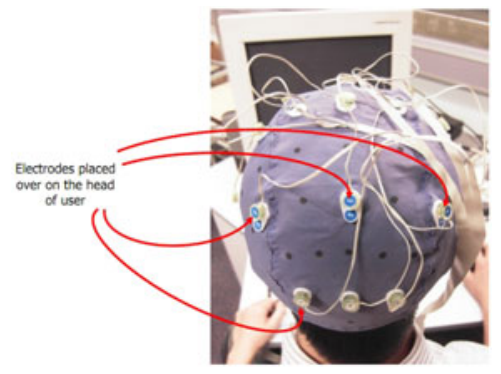

(b) EEG electrodes layout

Fig. 2. One example of the paragraphs and The EEG electrodes

each participant. After reading through 10 paragraph (a trial) - the recording is stopped and stored for offline analysis. Timestamps are marked to indicate the start and end of each reading of a paragraph.

\subsection{Signal Processing}

The EEG signals were originally collected at $1024 \mathrm{~Hz}$, which in turn were downsampled to a rate of 256 samples per second. We ran a low-pass filter of $60 \mathrm{~Hz}$ 
to eliminate unwanted EEG frequencies. The time-domain EEG signal is then be broken into epochs (windows) of one second. Fourier-Transformation (FFT) is performed on each window - for each of the 16 channels. The data from the FFT is binned into four frequency ranges: Delta, $(0-4 \mathrm{~Hz})$, Theta $(4-8 \mathrm{~Hz})$, Alpha $(8-13 \mathrm{~Hz})$ and Beta $(13-30 \mathrm{~Hz})$. Figure 3(b) show the output of FFT with the color-coded ranges. A peak detector (sensitivity of 0.7 ) is run on the FFT bins

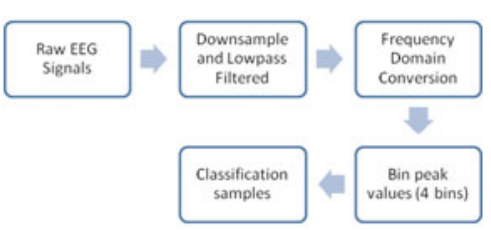

(a) Signal processing process

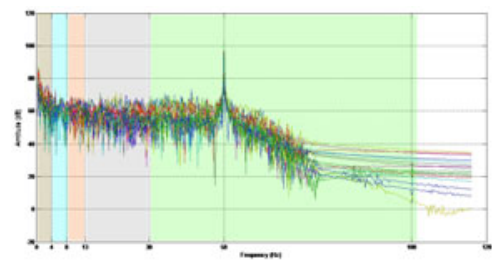

(b) visualisation of an FFT transformed for 16 channels of an epoch

Fig. 3. Signal Processing Blocks and FFT transformation

to detect peak values for each EEG band. Since we are interested in the activities of the four EEG bands (Alpha, Beta, Theta and Gamma), a window (epoch) will have four features representing it. Since we have 16 channels, our regular dataset will have 64 features ( $4 \times 16$ ). The same process $3(\mathrm{a})$, will be applied on the subsequence epochs until the end of EEG recording. The samples are labeled " 1 " to indicate reading relevant text against " 0 " for reading irrelevant text. We choose FFT because it is a very efficient transformation algorithm. Using it results in a signal processing capable of perform live (real-time) EEG signal processing. Optimization of window size could further improve the performance of this model.

\subsection{Classification}

Artifical Neural Network. With the dataset we captured, we think it will be sufficient for us to utilize a standard ANN configuration. The ANN setup we constructed for this experiment is a feed-forward, back-propagation network. This network has one hidden layer containing 20 hidden neurons and one output. As for the neural network optimization algorithm, we took the advantage of the Levenberg-Marquardt optimization (ML) training algorithm. The output value is described as [3], [6], [5]:

$$
y_{1}^{T}=g_{O}\left(b_{1}+\sum_{j} W_{1 j} \cdot g_{H}\left(b_{j}+\sum_{k} w_{j k} \cdot x_{k}^{T}\right)\right),
$$

The back-propagation training algorithm, being Levenberg-Marquardt optimization, will be represented by the formula [3] [6]:

$$
\delta w=\left(J^{T} J+I \cdot \mu\right)^{-1} J^{T} e
$$


The training performance is calculated using the Mean Square Error function. The training process is expected to stop once the performance (error) is minimized to the goal.

\section{Evaluation and Comparison}

\subsection{For Individual Participants}

We divided our dataset into smaller groups by participant - we called them P1, P2 all the way to P19. The average sample size of reach group is about 57 samples. For each group, we ran our constructed ANN with 10-Fold crossvalidation. The results are shown in table 1.

Table 1. ANN classification results for 19 participants (individual)

\begin{tabular}{|l|c|c|c||c|c|c|c|}
\hline & Accuracy & Specificity & Sensitivity & & Accuracy & Specificity & Sensitivity \\
\hline P1 & 0.928 & 0.966 & 0.872 & P11 & 1.000 & 1.000 & 1.000 \\
\hline P2 & 0.918 & 0.891 & 0.963 & $\mathbf{P 1 2}$ & 0.927 & 1.000 & 0.833 \\
\hline P3 & 0.986 & 0.977 & 1.000 & P13 & 0.968 & 1.000 & 0.920 \\
\hline P4 & 0.924 & 0.921 & 0.929 & $\mathbf{P 1 4}$ & 1.000 & 1.000 & 1.000 \\
\hline P5 & 0.781 & 0.667 & 0.929 & $\mathbf{P 1 5}$ & 1.000 & 1.000 & 1.000 \\
\hline P6 & 0.931 & 0.889 & 1.000 & P16 & 0.972 & 1.000 & 0.941 \\
\hline P7 & 0.984 & 1.000 & 0.958 & P17 & 0.966 & 0.962 & 0.972 \\
\hline P8 & 1.000 & 1.000 & 1.000 & $\mathbf{P 1 8}$ & 1.000 & 1.000 & 1.000 \\
\hline P9 & 0.900 & 0.871 & 0.947 & P19 & 0.985 & 1.000 & 0.963 \\
\hline P10 & 0.979 & 1.000 & 0.944 & Average & 0.955 & 0.955 & 0.956 \\
\hline
\end{tabular}

The results table above demonstrates the effectiveness of our method (data processing and classification). The average accuracy rate is about 95 percent, which is quite encouraging for the task of classifying EEG signals. There is still minor inconsistency in the achieved results - with P5 achiving about high $70 \%$ accuracies. It indicates that further studies could be done to investigate the profiles of these participants.

In relation to a real-time system for predicting this kind of scenario, we show that even with a relatively small effort of training i.e. reading tasks of only 10 paragraphs, we can still achieve a quite successful classification result. The potential for a working system based around this experiment is very promising.

\subsection{For the Whole Dataset}

This section explains our initial attempts in this work i.e. to actively identify an EEG pattern for the particular scenario - regardless of the individuals from whom we collected the EEG signal. For that purpose, we classified the whole dataset with the same ANN setup. This is to confirm our hypothesis that there 
Table 2. ANN classification results for 19 participants (together)

\begin{tabular}{|c|c|c|c|}
\hline Method & Accuracy & Specificity & Sensitivity \\
\hline EEG & $\mathbf{0 . 8 1 7}$ & 0.889 & 0.716 \\
\hline Gazetrack & $\mathbf{0 . 8 2 8 3}$ & 0.8687 & 0.7879 \\
\hline
\end{tabular}

is a general" EEG pattern. Similarly, we validated the results with 10 -Fold cross validation.

In this section, we also compare this result with EEG with the results we achieved with gaze-tracking data collected from the previous experiment [6]. The comparison is made with the result of Group B dataset where the experiment setup is almost identical to this experiment. The results are as followed

The results we got show that the overall dataset provides lower accuracy than the individual dataset. This is expected because EEG signals are variable or subjective in a number of aspects (time, person to person, mood, etc). We can also see that the accuracy we obtain with EEG is almost on par with the one obtained from gaze-tracking devices. This is very encouraging.

The accuracy we got here also suggests that by increasing the amount of training together with further optimization of ANN configurations, we could improve it to a more desirable level. This result has laid the foundation for further research work in this area.

\section{Conclusion}

This paper exhibits the capability of using EEG and statistical machine learning algorithms to distinguish different types of human brain activities. The results of this study, from the preliminary analysis stage throughout the final results have shown that there are certain relationships between EEG signals captured from the human brain to the way a person reads or perceives the information while reading. It may not give the definite answer to our hypotheses; and still suggests improvement could be made toward confirming them.

The scenarios we defined in this paper maybe a bit unnatural and forced, but it shows that we are in the right direction on the quest to establish a better model of the thinking brain from the BCI perspective.

\section{References}

1. Zhu, D., Gedeon, T., Taylor, K.: Keyboard before Head Tracking Depresses User Success in Remote Camera Control. In: Gross, T., Gulliksen, J., Kotzé, P., Oestreicher, L., Palanque, P., Prates, R.O., Winckler, M. (eds.) INTERACT 2009. LNCS, vol. 5727, pp. 319-331. Springer, Heidelberg (2009)

2. Byvatov, E., Fechner, U., Sadowski, J., Schneider, G.: Comparison of Support Vector Machine and Artificial Neural Network Systems for Drug/Nondrug Classification. Journal of Chemical Information and Computer Sciences, 1882-1889 (2003) 
3. Hagan, M.T., Menhaj, M.B.: Training feedforward networks with the Marquardt algorithm. IEEE Transactions on Neural Networks 5(6), 989-993 (1994), doi:10.1109/72.329697

4. Miller, G.A.: The Magical Number Seven, Plus or Minus Two: Some Limits on Our Capacity for Processing Information. In: The Psychology of Communication: Seven Essays. Penguin Books, Inc. (1970)

5. Mendis, B.S.U., Gedeon, T.D., Koczy, L.T.: Learning Generalized Weighted Relevance Aggregation Operators Using Levenberg-Marquardt Method. In: International Conference on Hybrid Intelligent Systems (2006)

6. Vo, T., Mendis, B.S.U., Gedeon, T.: Gaze Pattern and Reading Comprehension. In: Wong, K.W., Mendis, B.S.U., Bouzerdoum, A. (eds.) ICONIP 2010, Part II. LNCS, vol. 6444, pp. 124-131. Springer, Heidelberg (2010)

7. Makeig, S., Debener, S., Onton, J., Delorme, A.: Mining event-related brain dynamics. Trends in Cognitive Science 8, 204-210 (2004); Summarizes benefits and pitfalls of combining ICA, time/frequency analysis, and ERP-image visualization. (2004)

8. Hallez, H., et al.: Muscle and eye movement artifact removal prior to EEG source localization. In: Conference Proceedings of the International Conference of IEEE Engineering in Medicine and Biology Society, vol. 1, pp. 1002-1005 (2006) 